\title{
Análisis Espacial de la Segregación Residencial de Adultos Mayores en la Zona Metropolitana del Valle de Toluca
}

\author{
Spatial Analysis of Residential Segregation Older Adults in the Metropolitan \\ Zone Valley Toluca
}

\begin{abstract}
Alfredo Corona Consuelo ${ }^{1}$
Carlos Garrocho Rangel ${ }^{2}$

Juan Campos Alanís ${ }^{3}$
\end{abstract}

Recibido: 02 de junio de 2016

Aceptado: 02 de diciembre de 2016

\section{Resumen}

Este estudio se realiza dentro de la Geografía Gerontológica y propone un análisis para conocer el movimiento que efectúa la población adulta mayor en el interior de la Zona Metropolitana del Valle de Toluca (ZMVT), al segregarse residencialmente y aglomerarse en un grupo donde la población vecina cumple las mismas características socioresidenciales. La investigación se basa en la aplicación de dos métodos geoeconométricos: los índices aespaciales y los índices espaciales, utilizando los software de libre acceso GEODA y Geo Segregation Analyzer, con el propósito de argumentar cuál de los métodos es de mayor utilidad en los problemas socioespaciales. El resultado demuestra que la población adulta mayor se ubica en el centro de la ZMVT, patrón que se cumple para todos los centros de población de los municipios que conforman esta ZMVT, siendo las nuevas zonas habitacionales el lugar de residencia para las nuevas familias o población relativamente joven. Se concluye que los índices espaciales mediante la aplicación de la autocorrelación espacial y el Índice Local de Moran son los que arrojan resultados más exactos, precisos y localizables, por lo que serían de mayor utilidad en el análisis de este tipo de problema.

Palabras clave: autocorrelación espacial, geoeconometría, Geografía Gerontológica, Índice Local de Moran, segregación residencial.

\begin{abstract}
This study is performed within of the Gerontological Geography, as analysis in order to know the movement that the older population effected within the ZMVT segregated residentially and agglomerate in a group where the neighboring town meets the same socio-residential properties. It is based on the application of two methods Geoeconometrics; Aespaciales indexes and spatial indexes, using the software's of freely available GEODA and Geo Segregation Analyzer, this in order to argue, which method is most useful in the socio-spatial problems. The result shows that the older population is located in the center of the ZMVT. This pattern holds for all population centers of the neighboring municipalities of this ZM, being the new residential areas (regular and irregular) and the residential complexes the place of residence for new families or relatively young population. Likewise spatial indexes by applying spatial autocorrelation and Local Moran Index are what give more accurate, precise and localized results, so these are most useful in the analysis of this type of problem.
\end{abstract}

Keywords: geoeconometrics, geography gerontology, Local Moran index, residential segregation, spatial autocorrelation.

\footnotetext{
${ }^{1}$ Facultad de Planeación Urbana y Rural, Universidad Autónoma del Estado de México. Contacto: alfcorona@itecel.com

${ }^{2}$ Colegio Mexiquense. Contacto: cfgarrocho@gmail.com

3 Facultad de Geografía, Universidad Autónoma del Estado de México. Contacto: juan_campos70@gmail.com
} 


\section{Introducción}

El envejecimiento poblacional comprende un cambio en la estructura por edad y se manifiesta en el aumento del porcentaje de personas en edad avanzada. Según el Instituto Nacional de Geografía, INEGI (2010), las proyecciones realizadas por el Fondo de Población de Naciones Unidas (UNFPA, por sus siglas en inglés), muestran que en 2050 uno de cada cinco habitantes en el planeta $(21,2 \%)$ tendrá 60 años o más. En las regiones menos desarrolladas, una de cada tres personas (32\%) será un adulto mayor. Estos datos indican que en el futuro mediato, el incremento de personas de edad avanzada puede convertirse en un serio problema económico, social y de salud pública, ya que se requerirán fondos públicos para cubrir la demanda de pensiones, mayor infraestructura (casas-habitación, calles y transporte público) y una mayor cobertura especializada de servicios de salud.

Como antecedente general, en 2010, se registraron en el Estado de México 1.137.647 habitantes de 60 años o más, equivalente al 7,4\% de la población y se espera que para 2030 se aproximen a los 3 millones de adultos mayores, los que representarían poco más de $16 \%$ de la población total de la entidad (INEGI, 2010). La Zona Metropolitana del Valle de Toluca (ZMVT) presenta cifras ligeramente inferiores a las del estado en su totalidad. En 2010, los 15 municipios que conforman la ZMVT concentraron un total de población de 1.936.126 habitantes (INEGI, 2010), de los cuales 83.195 habitantes eran mayores de 60 años, lo que representaba un 4,9\% del total de población; se prevé que para 2030 este grupo de población aumente a 170.350 personas de más de 60 años (INEGI, 2010).

En este estudio se propone un análisis para conocer el movimiento que realiza la población adulta mayor en el interior de la ZMVT, para establecerse residencialmente y aglomerarse en un grupo donde la población vecina cumple las mismas características socioresidenciales. La investigación se llevó a cabo mediante la utilización de dos métodos: los de índices aespaciales y los índices espaciales, con el fin de identificar cuál de los dos métodos es de mayor utilidad en los problemas socioespaciales.

En un país como México, que vive un proceso de envejecimiento acelerado de su población, la edad resalta como un factor de segregación. Por ello, el presente artículo postula la siguiente hipótesis: a mayor segregación residencial de la población adulta mayor en la ZMVT, mayor aislamiento y vulnerabilidad de este grupo (Garrocho y Campos, 2005). La pregunta central que emerge de esta conjetura es la siguiente: ¿Cuál es la situación actual de la ZMVT en lo que respecta a la segregación residencial de su población adulta mayor? Responder a este cuestionamiento implica responder otras varias interrogantes, como por ejemplo ¿cuál es la intensidad de la segregación residencial? y ¿cuál es su patrón espacial? 
El resultado de este trabajo demuestra que la población adulta mayor se ubica en el centro de la ZMVT. Este patrón se cumple para todos los centros de población de los municipios aledaños que conforman esta ZMVT, siendo las nuevas zonas habitacionales (regulares e irregulares) y conjuntos habitacionales los lugares de residencia para las nuevas familias o población relativamente joven. Los índices espaciales son los que dan resultados más exactos, precisos y localizados, por lo que estos son de mayor utilidad en el análisis de este tipo de problema.

\section{Argumentos conceptuales}

Para poder realizar el análisis geoeconométrico de fenómenos geográficos (para este caso, de Geografía Gerontológica), se requiere de los siguientes agentes indispensables:

Agentes de exposición. En los siguientes puntos se revisan estos elementos: dato espacial, Geografía Gerontológica, segregación residencial y autocorrelación espacial.

Dato espacial. Según su descripción, un dato espacial puede definirse como la observación de una variable asociada a una localización del espacio geográfico, es decir, es un fenómeno que se observa y ocurre en un lugar específico y que, además, tiene localización geográfica. También se puede establecer que un dato espacial es una variable asociada a una localización del espacio. Normalmente, se utilizan datos vectoriales, los cuales pueden ser expresados mediante tres tipos de objetos espaciales.

Geografía Gerontológica. En el inicio de este siglo, el envejecimiento de la población se convierte en el fenómeno demográfico más importante que vive México (Ordorica, 2012). Desde los años 50, la mayoría de los países disminuyó en diferente grado su nivel de fecundidad $y$, por consiguiente, aumentó su sobrevivencia, tendencias que han incidido en el proceso de envejecimiento poblacional y que caracteriza a gran parte de los países del mundo.

Ahora bien, para definir cómo se es una persona adulta mayor, es necesario abordar el concepto desde una perspectiva biopsicosocial o también llamada visión integral. Al respecto, la dimensión urbana del envejecimiento es altamente relevante, porque las ciudades concentrarán de manera creciente la población del país, incluyendo a la población envejecida (Garrocho, 2013).

Desde el punto de vista del Instituto Nacional de Geriatría (ING, 2014 en Corona, Garrocho y Campos, 2014), el envejecimiento es un proceso de cambios a través del tiempo, gradual, continuo, irreversible y completo. Además, estos cambios se dan a nivel biológico, psicológico y social, y son determinados por la historia, la cultura y la situación económica, tanto de los 
grupos como de las personas. Así mismo, este instituto señala que cada ser humano envejece de manera diferente, lo cual depende de las características innatas, de las que adquiere a través de la experiencia y de las circunstancias a las que se haya enfrentado durante su vida (Corona et al., 2014).

Segregación residencial. En principio, la segregación se refiere a la acción de separar o apartar una unidad del resto (Prieto, 2010). Rodríguez (2008a) subraya que la segregación residencial está registrando incrementos notables; en tanto que Borja y Castells (2000) indican que esta es originada por la mezcla de factores:

- De nivel mundial, a partir de los años 80 se registran cambios estructurales (globalización, creciente acción de los mercados y desregulación), que benefician a algunos segmentos de la población y perjudican a otros, además de originar un aumento de las disparidades socioeconómicas.

- Las tendencias hacia la liberalización de los mercados de tierras favorecen un vínculo mucho más fuerte entre el valor del suelo y el nivel socioeconómico de la población.

- El aumento de las condiciones de inseguridad en las ciudades genera la búsqueda de lugares protegidos por parte de la población con mayores recursos.

- El surgimiento de áreas cada vez más protegidas y seguras, con presunciones de exclusividad de los grupos socialmente emergentes.

- Los aspectos regresivos (a escala local) de la descentralización, en particular la relación directa entre recursos disponibles por los gobiernos locales y el nivel socioeconómico de sus residentes, que lleva a una inversión municipal per cápita más alta en lugares en donde residen los grupos de mayores ingresos.

Entonces, la segregación residencial se refiere a la separación en el territorio de diferentes grupos de población. Se dice que un grupo es segregado espacialmente cuando sus integrantes no se distribuyen de manera homogénea en el territorio en relación con el resto de la población (Garrocho y Campos, 2005). Del mismo modo, Checa-Olmos y Arjona Garrido (2007), manifiestan que la segregación puede ser entendida como una separación residencial de distintos grupos poblacionales. Expresado de otra manera, se puede decir que la segregación espacial existe cuando ciertas áreas muestran una sobrerrepresentación de un grupo poblacional, mientras que otras registran una sobrerrepresentación del mismo grupo.

Como se aprecia, la segregación residencial, entendida como la manifestación espacial del aislamiento, separación o distanciamiento de un grupo poblacional de otros grupos de población de un determinado espacio geográfico, responde a un conjunto de factores económicos, sociales, demográficos y culturales (Ortiz y Morales, 2002; Rodríguez, 2008b). 
Autocorrelación espacial. La herramienta Índice de Moran mide la autocorrelación espacial basada en las ubicaciones y los valores de las entidades simultáneamente. De acuerdo con un conjunto de entidades y un atributo asociado, evalúa si el patrón expresado está agrupado, disperso o es aleatorio. La herramienta calcula el valor del Índice I de Moran, una puntuación z y un valor $\mathrm{P}$ para evaluar la significancia de ese índice. Los valores $\mathrm{P}$ son aproximaciones numéricas del área debajo de la curva de una distribución conocida, limitada por la estadística de prueba (Anselin, 1995; Moreno y Vayá, 2000).

Esta es una herramienta de estadística deductiva, lo que significa que los resultados del análisis siempre se interpretan dentro del contexto de la hipótesis nula.

Para la estadística I de Moran global, la hipótesis nula establece que el atributo que se analiza está distribuido en forma aleatoria entre las entidades del área de estudio, es decir, los procesos espaciales que promueven el patrón de valores observado constituyen una opción aleatoria. Usualmente, en el análisis de fenómenos económicos espaciales por medio del estudio econométrico suelen surgir dos problemas que son inherentes a la naturaleza propia de la estructura de los datos a analizar: la heterogeneidad espacial y la autocorrelación espacial o dependencia espacial (Anselin, 1995; Moreno y Vayá, 2000).

La dependencia espacial significa que los valores de una misma variable, que son medidos en localidades que son cercanas entre sí, tienden a ser similares, es decir, a mayor cercanía geográfica corresponde una mayor similitud en los valores. La dependencia espacial se produce, entonces, cuando el valor de la variable dependiente en una unidad espacial funge como el valor de la misma variable en unidades vecinas. La variable de dependencia espacial hace diferente la estadística espacial de la tradicional, debido a que considera la distancia y la contigüidad existentes en las unidades de observación (Moreno y Vayá, 2000).

Ahora bien, la heterogeneidad espacial refiere a la idea de diferenciación geográfica en las relaciones que comprenden los fenómenos económicos espaciales, conforme varía el espacio de estudio.

La heterogeneidad espacial o heterocedasticidad espacial es frecuente en estudios económicos que tienden a estudiar un fenómeno determinado con la orientación centro-periferia, ya que cada región deriva en diferentes valores de las medidas.

Según Anselin (1995) y Moreno y Vayá, (2000), la metodología que se aplica para solucionar la dependencia espacial debe ser diferente de la aplicada para la dependencia de modelos temporales, ya que estas series son 
unidireccionales (el pasado afecta al presente) en tanto que la dependencia espacial es multidireccional (ya que una región pudiera verse afectada por regiones vecinas, pero de igual manera influir sobre las mismas).

\section{Argumentos metodológicos: Software para la Autocorrelación Espacial con Índice Local de Moran ó LISA (Local Spatial Autocorrelation)}

¿Cómo conseguir el software GEODA?. Se trata de un software de distribución gratuita y para acceder a él se debe ingresar a un vínculo de la Universidad de Arizona y, previa inscripción, se puede descargar. Los detalles de la aplicación del software se pueden revisar en la realización paso a paso del mismo programa. Por último, una vez que se ha descargado el programa, se debe ejecutar el archivo OpenGeoda.exe para la instalación y, por último, se ingresa al programa en el ícono de GEODA.

Instrucciones para replicar el ILM (LISA). De acuerdo con el trabajo de Anselin, Rey y Li (2014), para iniciar se debe seleccionar el mapa respectivo, el cual debe tener una extensión .shp y debe contener asociados los datos de interés.

Para esto se sigue a continuación File, Open Shapefile y se localiza el archivo manzanas.shp; al hacer clic en abrir se observa el mapa de mapas censales de la Zona Metropolitana del Valle de Toluca (Anselin et al., 2014; Garrocho y Campos, 2005).

Para ver la información que contiene el archivo en la tabla manzanas.dbf, se debe hacer clic en open table. La información contenida en manzanas.dbf corresponde a los datos de la población (total, por género, por rangos de edad, por nivel educativo, etc.), obtenida del SCINCE del INEGI (2010).

Es importante hacer una revisión de la información contenida en la tabla de datos, a fin de ubicar la información de interés para que esta coincida, consultando el diccionario de datos, que en el caso de esta investigación se obtuvo de la página del INEGI y del SCINCE (Anselin et al., 2014).

Georreferenciar las variables a trabajar. Para llevar a cabo este paso se debe utilizar la herramienta Map. Para ello, se selecciona la opción Quantile map, indicando al proyecto la variable que se quiere georreferenciar, en este caso POB_24.

En el cuadro de diálogo subsiguiente se solicita ingresar la cantidad de clases en las que se va a dividir la información de la variable seleccionada (se privilegia la que ofrece por default el programa, esto es, 4 clases). 
Esta configuración realiza el gráfico para los cuantiles de la variable POB_24. Las zonas de color más intenso se refieren a los lugares donde la variable registra valores más altos. De esta misma forma se trabaja con los demás mapas.

Por su parte, el mapa de desviación estándar (Standard deviation map), presenta los estados con mayor variabilidad.

La construcción de ponderaciones espaciales. Haciendo una analogía con el texto de Anselin y colaboradores (2014), el primer paso en el análisis de autocorrelación espacial es construir un archivo de ponderaciones espaciales que contenga información respecto de la estructura municipio para cada ubicación. GEODA tiene muchas maneras de crear pesos espaciales.

Para iniciar el proceso de selección de pesos estos se deben crear en el menú Herramientas, o bien, se hace clic en el botón Crear Pesos, de la barra de herramientas misma. Hay que tener en cuenta que el menú Herramientas también puede desplegarse sin tener un proyecto abierto, lo que permite la creación de archivos de pesos para su uso en programas diferentes de GEODA.

En esta sección aparece un cuadro de diálogo que dice Create weights (Creación de pesos). Este contiene todo acerca de las opciones disponibles en GEODA. Se deben tener en consideración las tres partes en el diálogo. La parte superior requiere de la especificación del archivo de entrada (un archivo de forma); el archivo de salida (archivo de ponderaciones espaciales), y una variable de cada peso de identificación exclusiva (Anselin et al., 2014). El identificador se usa para asegurar que las entradas en el archivo de pesos coinciden con las entradas apropiadas en la base de datos.

Por otro lado, la parte media del cuadro de diálogo se refiere a los pesos de contigüidad. Se pueden construir dos tipos de contigüidad Rook (Torre) y Queen (Reina), así como archivos que contienen contigüidad en orden superior. La parte inferior de los diálogos construidos con la distancia se basa en pesos espaciales de coordenadas $x y$. La malla corresponde a las coordenadas en un archivo de forma o elemento $x y$, las variables contiene el conjunto de datos.

Para crear un archivo de pesos de contigüidad de torre, dependiente de la incorporación de los municipios de la ZMVT, primero se debe hacer clic en el ícono de archivo abierto y seleccionar ZMVT.shp como archivo de entrada. A continuación, se hace clic en el ícono de guardar el archivo y se escribe un nombre para el archivo de pesos, por ejemplo PAM (el archivo obtendrá una extensión de archivo .gal).

En tercer lugar, se debe ingresar en la variable de identificación y el registro de la variable y el tipo de contigüidad. Por último, se hace clic en Crear, para 
iniciar el proceso: aparecerá una barra de progreso indicado cuando haya sido creado el archivo. Los pesos aparecerán en el archivo en el directorio de trabajo actual y a partir de ese momento están disponibles para su uso en el análisis.

Ponderaciones espaciales (características). Por último, antes de ingresar en el cálculo de las estadísticas de autocorrelación espacial, una buena práctica es comprobar los pesos espaciales de la presencia de islas (observaciones inconexas) y otras características indeseables.

Un histograma con la distribución del número de vecinos de un archivo de pesos dado se obtiene seleccionando Herramientas> Pesas > Propiedades, 0 bien, haciendo clic en el botón de la barra de Herramientas que dice Características de pesos. Esto exhorta un nuevo diálogo en el que se deberá especificar el archivo de pesos.

\section{Caso de estudio: análisis y resultados de segregación residencial de adultos mayores (SRAM) en la ZMVT}

Se estima que en las siguientes tres décadas la población menor de 15 años disminuirá y la proporcional a la población mayor de 65 años se incrementará, por lo que el rango de población de 15 a 64 años aumentará su importancia.

Según el Consejo Nacional de Población, CONAPO (2013) estas condiciones demográficas constituyen el llamado bono demográfico, lo cual propone oportunidades para invertir en capital humano para el desarrollo y hacer frente al envejecimiento demográfico, lo que debiera aprovecharse para estimular el ahorro interno, efectuar inversiones en salud, educación y capacitación laboral. Ello, debido a que según las proyecciones de CONAPO para el 2020 habría alrededor de 82,6 millones de adultos mayores de 65 años y para 2050, la cifra crecería a 85,5 millones, con lo cual la población mexicana completaría la fase final de transición demográfica orientándose hacia un crecimiento reducido y un perfil envejecido.

De acuerdo con dichas proyecciones, la edad media pasará de 29 años en 2010 a 31 en 2020 y a 38 en 2050, en tanto que la población menor de 15 años disminuirá de 33,9 millones en 2010 a 32,7 millones en 2020 y 28,9 millones en 2050; por último, el grupo de adultos mayores aumentará su tamaño de 7,1 millones en 2010 a 9,8 millones en 2020 y a 23,1 millones para 2050 (CONAPO, 2013).

Por otra parte, el envejecimiento demográfico involucra un cambio en la estructura por edad y generalmente se expresa en un aumento del porcentaje de personas en edad avanzada. En 2012, de acuerdo con el Fondo de Población de Naciones Unidas (UNFPA, por sus siglas en inglés), 11,5\% de la población 
mundial tenía una edad de 60 años y más, mientras que en las regiones más desarrolladas la cifra llegaba a $22,6 \%$. Proyecciones realizadas por el UNFPA, indican que en 2050, uno de cada cinco habitantes en el planeta $(21,2 \%)$ tendrá 60 años y más; en las regiones menos desarrolladas será de 19,5\%, es decir, casi el nivel que actualmente se observa en las regiones más desarrolladas, y en estas últimas, una de cada tres personas (32\%) será un adulto mayor en 2050 (INEGI, 2010). En México la participación relativa de adultos mayores ha pasado de $6,2 \%$ en 1990 a 9,3\% en 2012 y se espera que para el año 2050 sea de 21,5\%.

En esta línea, Según el INEGI (2010) los adultos mayores transitan por diversas etapas de desarrollo que marcan estilos de vida diferenciados, toda vez que se hace evidente la pérdida gradual de capacidades motrices y cognoscitivas conforme avanza la edad. Es así que de los 10,9 millones de personas de 60 años y más que en 2012 residían en el México, 31,1\% se encontraba en una etapa de prevejez (60 a 64 años); $41,3 \%$ estaba en una vejez funcional (65 a 74 años); $12,5 \%$ en una vejez plena ( 75 a 79 años) y $15,1 \%$ estaba transitando por una vejez avanzada (80 años y más).

En 2010 se registraron en el Estado de México 1.137.647 habitantes de 60 años a más lo que equivale al 7,4\% de la población y se espera que para 2030 se aproximen a los 3 millones de adultos mayores, con lo que abarcarían poco más de $16 \%$ de la población total de la entidad (INEGI, 2010).

Ahora bien, en relación con la segregación residencial de adultos mayores (SRAM) para la ZMVT y el trabajo con el software GEODA, en primera instancia se realizó un acercamiento con los datos a nivel de municipio, llegando a resultados muy generales, globales e integradores, donde en la ZMVT la SRAM se tiene en un rango de bajo-bajo (Low-Low) y el área más segregada para esta población fue la zona sur del estado, dentro del rango de alto-alto (HighHigh).

Cabe hacer mención que este programa tiene la facilidad de poder hacer filtros dentro de los resultados. Para la investigación se tomó en cuenta el nivel de significancia el rango de 99,95, o sea, que existe un ,05\% de error dentro de los resultados obtenidos a nivel de municipio.

En la Figura 1 se muestra el mapa de significancias, donde se pueden observar los municipios que caen dentro de cada uno de los errores tomados por el software y en los que se aplican los filtros. Cabe mencionar que el filtro no tiene mayor importancia, ya que de acuerdo con el error que se toma en cuenta, el número de municipios u objetos dentro del rango de No significantes (Not significants) va en aumento y disminuyen los rangos de ,05 \% de error y sucesivamente conforme se aplica el filtro. 

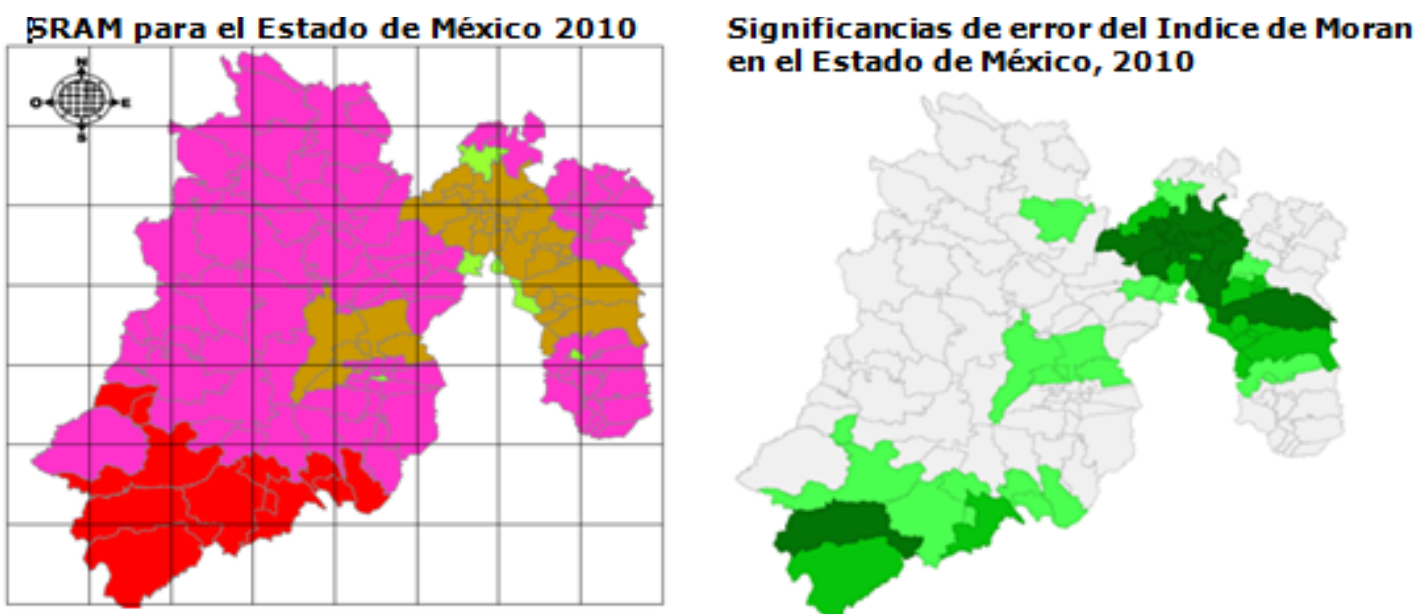

Diagrama de dispersión de Moran.
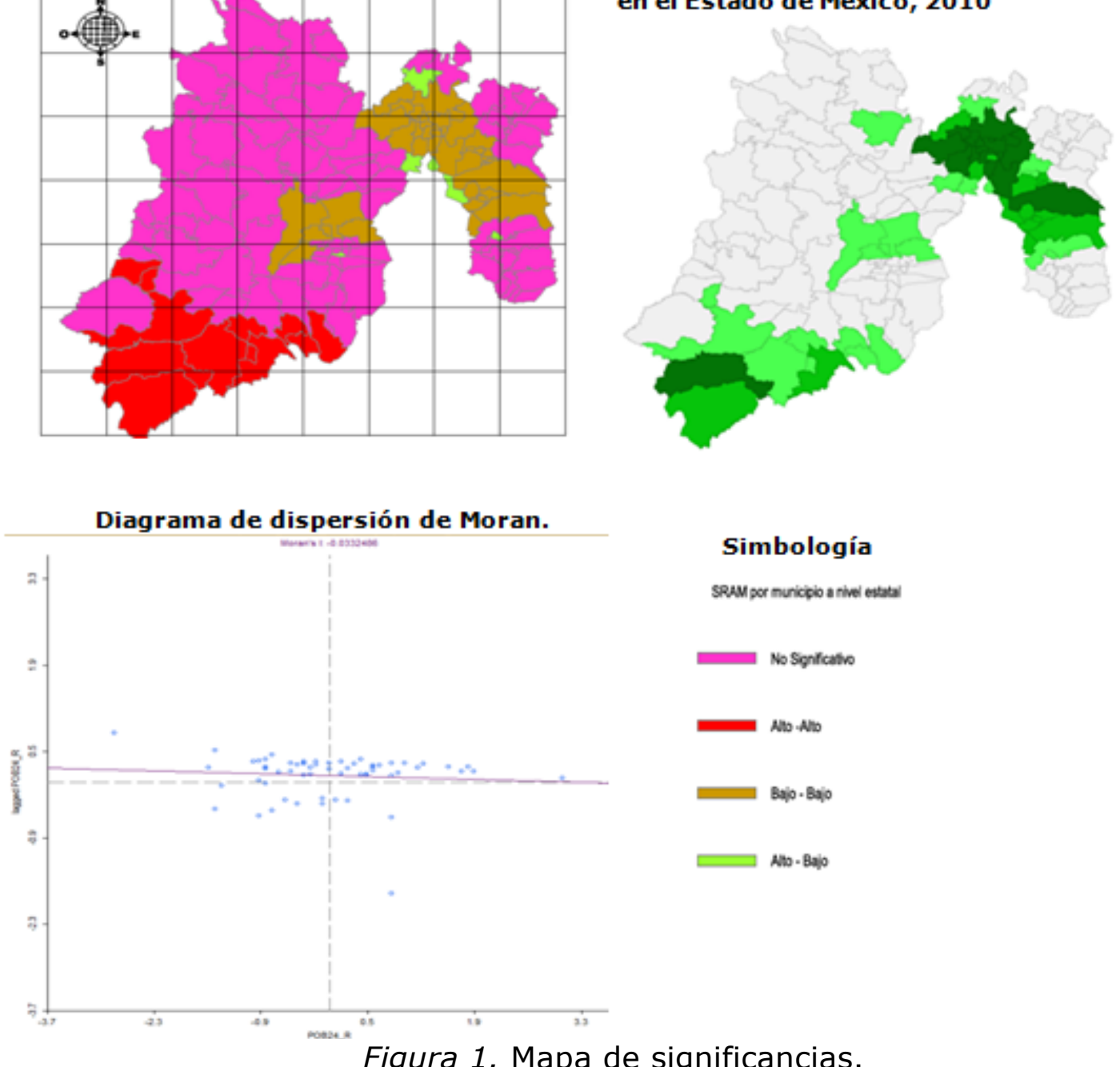

Figura 1. Mapa de significancias.

Fuente: Elaboración propia sobre la base de datos de SCINCE 2010 (INEGI) y con ayuda de software GEODA y gv SIG.

Por otra parte, en la Figura 2 se observa el grado de marginación que según los datos del Censo de Población y Vivienda del INEGI para el 2010 existían en la ZMVT y como se puede observar en la zona del centro de esta Zona Metropolitana el rango es Muy Bajo, por consiguiente del grado de urbanización e importancia económica que esta zona adquiere. En los AGEB's que comprenden los municipios que forman parte de esta Zona Metropolitana los rangos van desde Medio, Alto y hasta Muy Alto, cuestión que afecta a la población que conforma estas partes y que son los adultos mayores. 


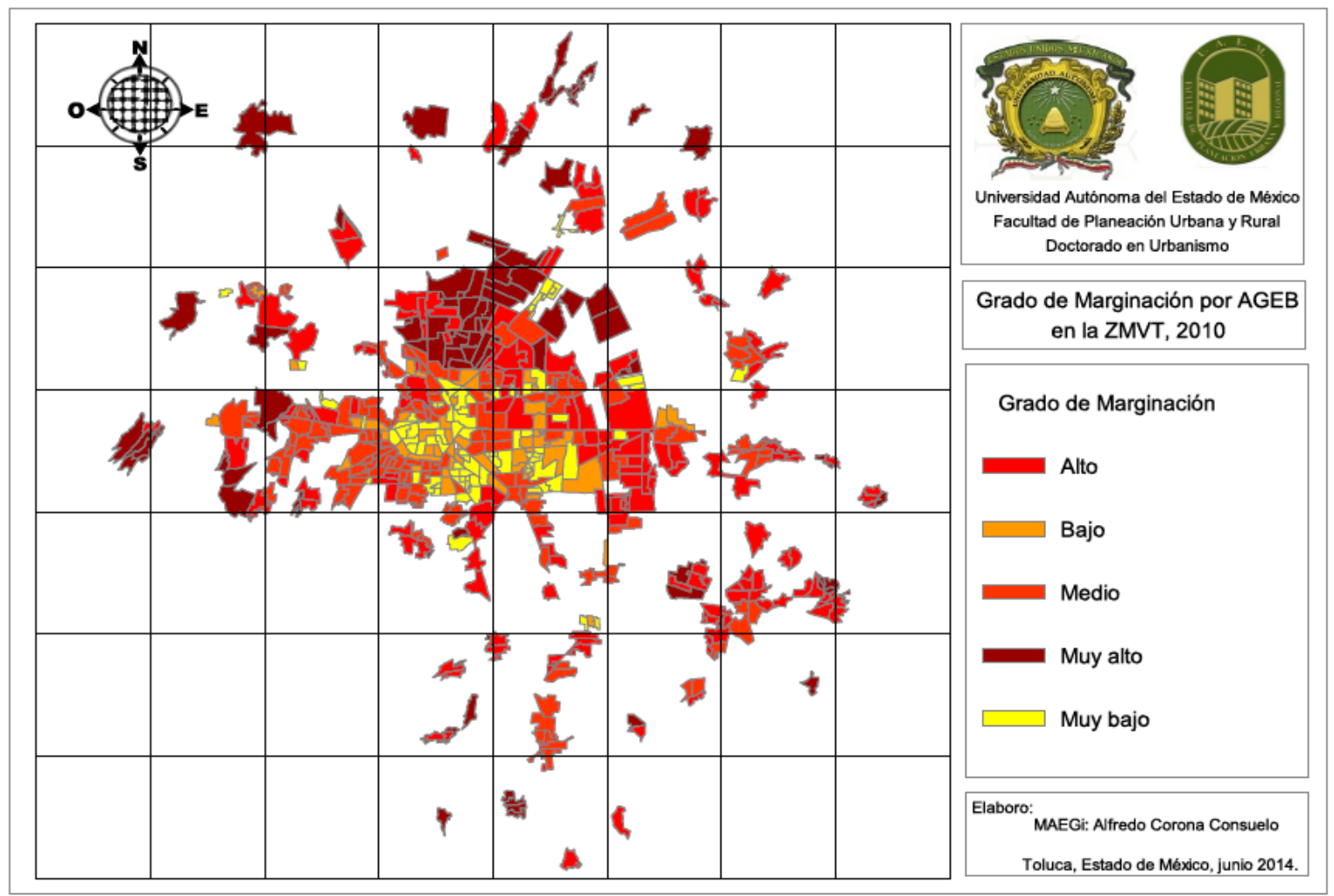

Figura 2. Mapa de grado de marginación por AGEB de ZMVT, 2010. Fuente: Elaboración propia sobre la base de datos de SCINCE 2010 (INEGI) y con ayuda de software GEODA y gv SIG.

En la Figura 3, en tanto, se puede constatar que según el análisis realizado por el software GEODA en el centro existe un alto índice de segregación residencial de adultos mayores y algunas islas con el rango de No Significativas. Esto no significa que no exista segregación, porque consecuentemente existe, solo que se trata de zonas céntricas o urbanas de los municipios aledaños o periféricas de esta gran ZMVT y, por la distancia que existe entre estas áreas y el centro de la ZM, sería esta la razón por el cual el software la considera como No Significativa. 


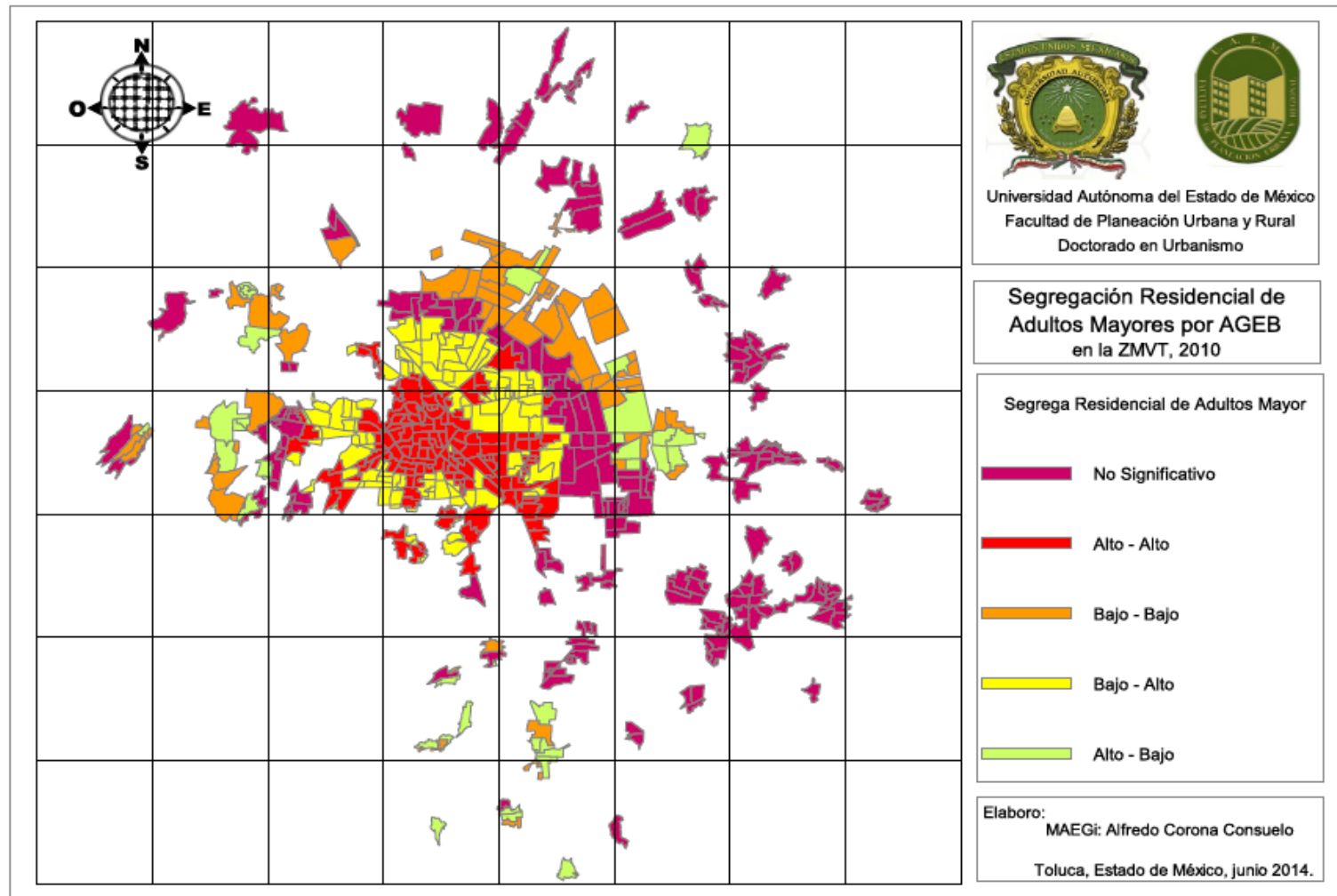

Figura 3. Mapa de SRAM en ZMVT, 2010.

Fuente: Elaboración propia sobre la base de datos de SCINCE 2010 (INEGI) y con ayuda de software GEODA y gv SIG.

Ahora bien, según el Índice de Fecundidad (Figura 4) también en el centro de las ciudades de Toluca y Metepec y, por consiguiente, en el centro de la Zona Metropolitana, incluyendo los municipios más cercanos aledaños a esta (Almoloya de Juárez, Lerma, San Mateo Atenco y Zinacantepec, principalmente) es donde este indicador ha disminuido, lo cual repercute en la edad de esta zona. 


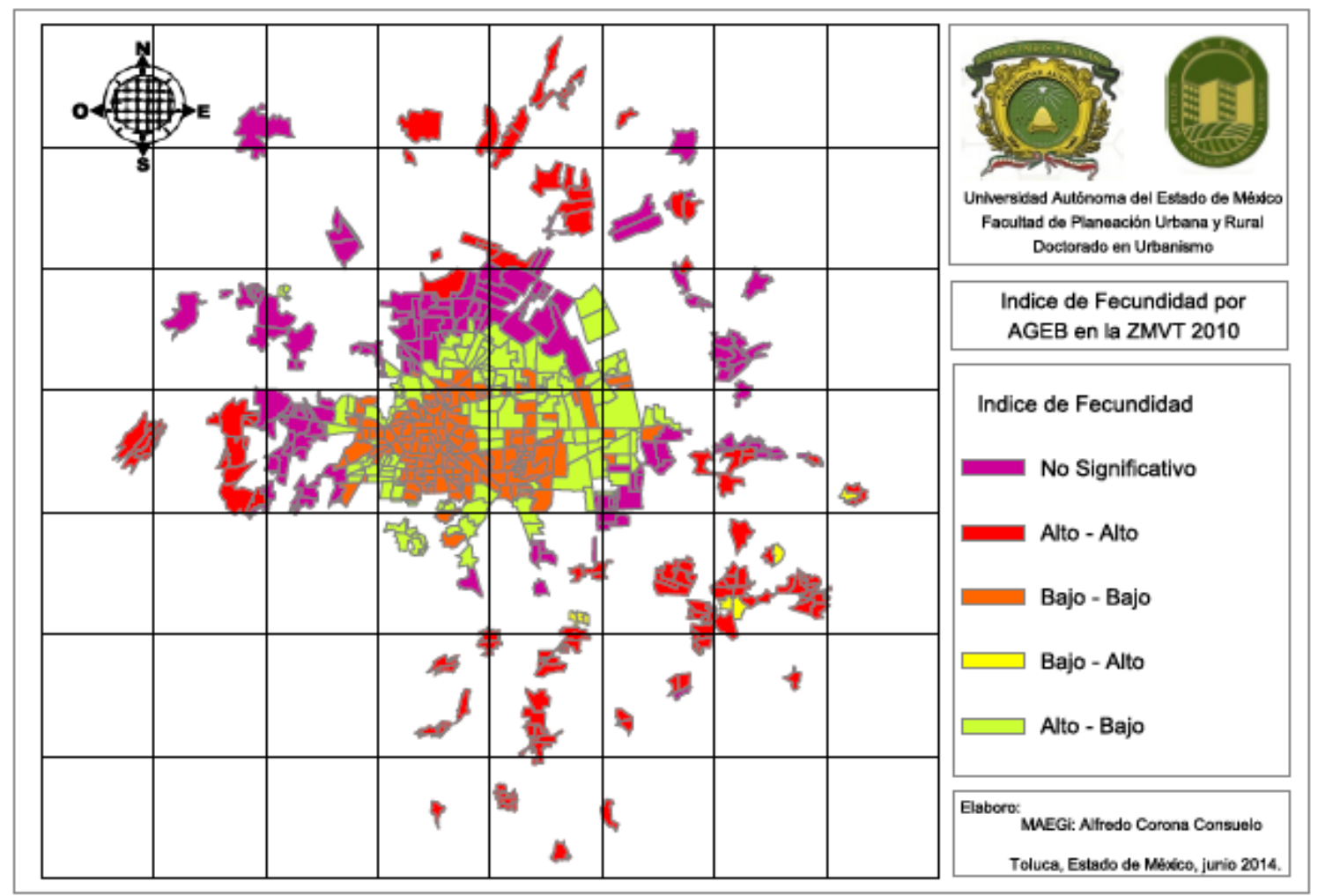

Figura 4. Mapa de Índice de Fecundidad.

Fuente: Elaboración propia sobre la base de datos de SCINCE 2010 (INEGI) y con ayuda de software GEODA y gv SIG.

De esta misma manera, en el mapa de Índice de Mortalidad (Figura 5) se puede apreciar que este índice interviene en las áreas antes mencionadas, donde también ha disminuido, por lo que ahí se encuentra segregada la población de adultos mayores. Simplemente en los conjuntos habitacionales se puede observar que ni existe SRAM, ni el Índice de Fecundidad disminuye y mucho menos el Índice de Mortalidad se ve disminuido, lo que da la visión de que es una población relativamente joven, conformada por parejas recientes o jóvenes que tienen entre 1 y 0 hijos, y que están dedicados a trabajar y desarrollarse profesionalmente. 


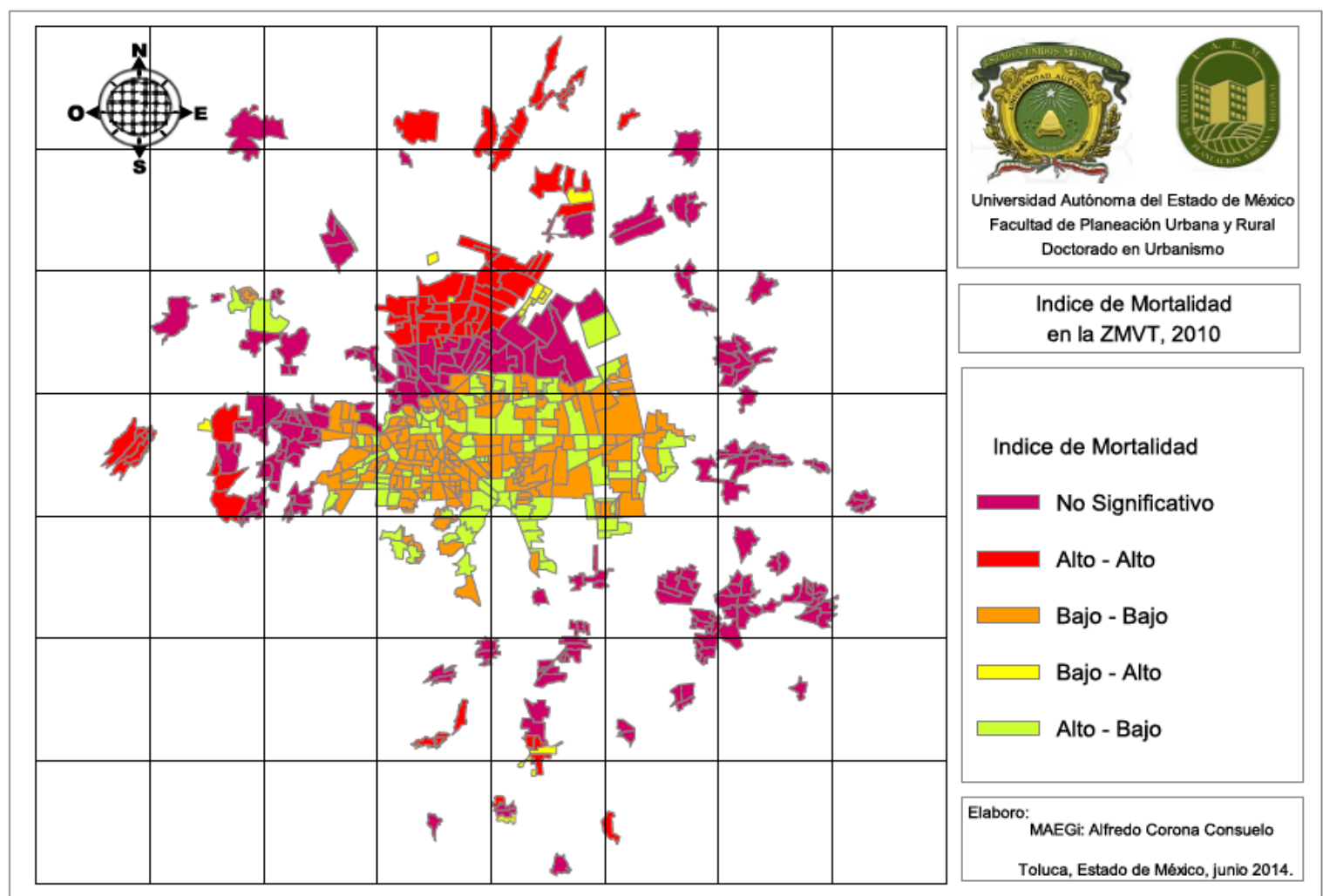

Figura 5. Mapa de Índice de Mortalidad, 2010.

Fuente: Elaboración propia sobre la base de datos de SCINCE 2010 (INEGI) y con ayuda de software GEODA y gv SIG.

SRAM en la ZMVT a gran escala (a nivel local o nivel manzana). El diagrama de dispersión de Moran dice que existe un gran esparcimiento de datos, principalmente por lo extenso de la Zona Metropolitana ${ }^{4}$.

Como puede observarse en la Figura 6 (con mejor visibilidad en Figuras 7 y 8 ), en general, todos los centros de urbanizados ya sea de pueblos (rango altoalto), o localidades que antes eran pueblos aislados, por el proceso de metropolización de la ciudad se han ido adhiriendo a esta Zona Metropolitana, donde reside la población adulta mayor que, por el simple rasgo cultural de que ellos son quienes poblaron dichos centros $y$, por consiguiente de la expansión demográfica, son los hijos de estas personas mayores quienes se establecieron en las áreas reservadas para cultivo, o que han sido urbanizadas por los promotores inmobiliarios legal o ilegalmente.

\footnotetext{
${ }^{4}$ Se están tomando todos los municipios que tiene en cuenta el Grupo de la Secretaría de Desarrollo Urbano y Vivienda del Estado de México, donde se incluyen siete más de la delimitación realizada por el Grupo Interinstitucional SEDESOL-CONAPO-INEGI.
} 
Del mismo modo, puede observarse que el rango naranja (bajo-bajo) corresponde a la mayoría de los fraccionamientos y conjuntos urbanos habitacionales creados en la periferia de la ciudad de Toluca que contribuyeron a la expansión de la Zona Metropolitana.

La confiabilidad que dan los resultados (Figura 6) es positiva: el mapa de significancias señala que en la zona verde obscura es donde existe mayor confiabilidad $(99,99 \%)$, en tanto que en el verde claro los datos tienen un $99,95 \%$ de confiabilidad.

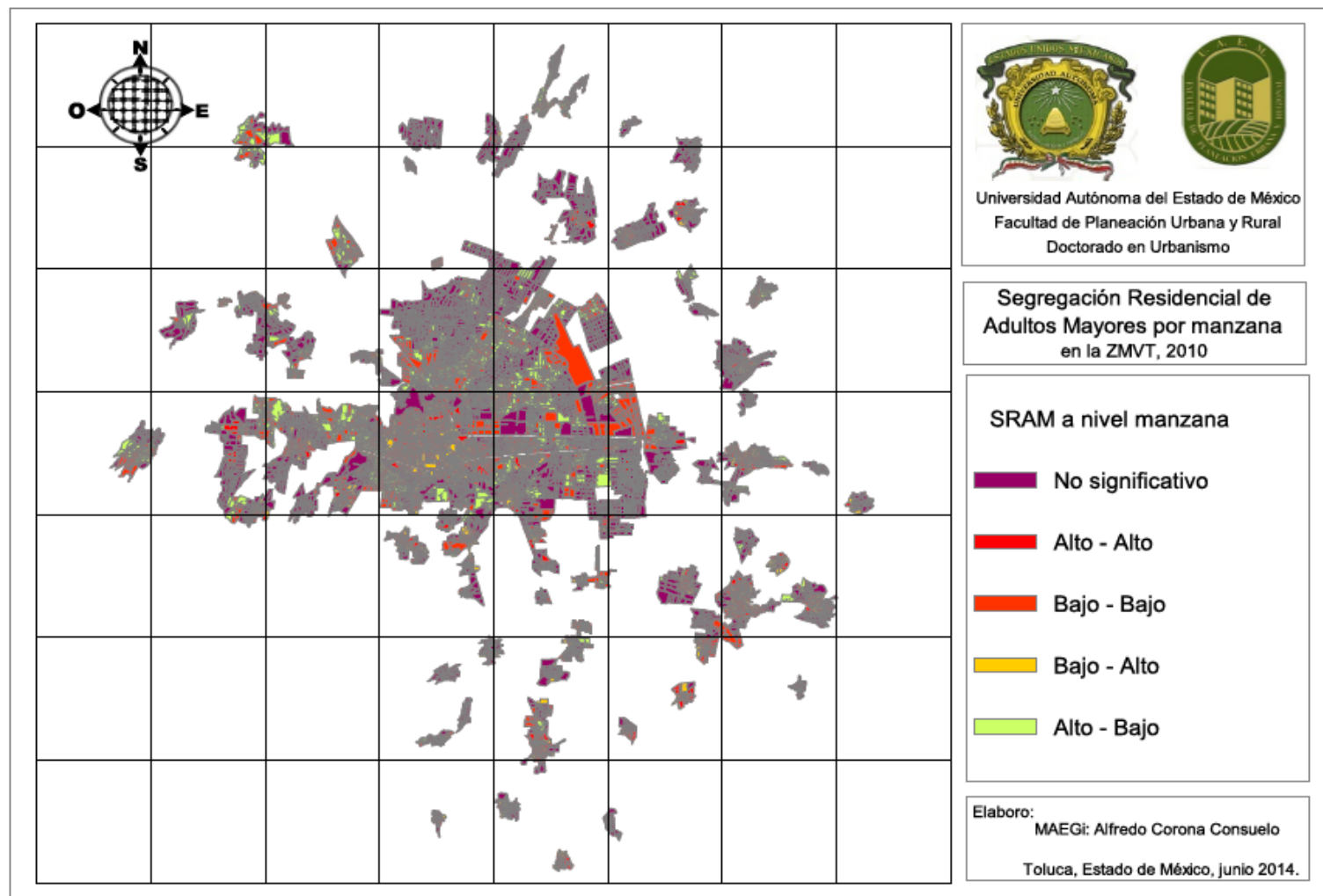

Figura 6. Mapa de Índice de Moran para la SRAM en ZMVT, 2010. Fuente: Elaboración propia sobre la base de datos de SCINCE 2010 (INEGI) y con ayuda de software GEODA y gv SIG. 


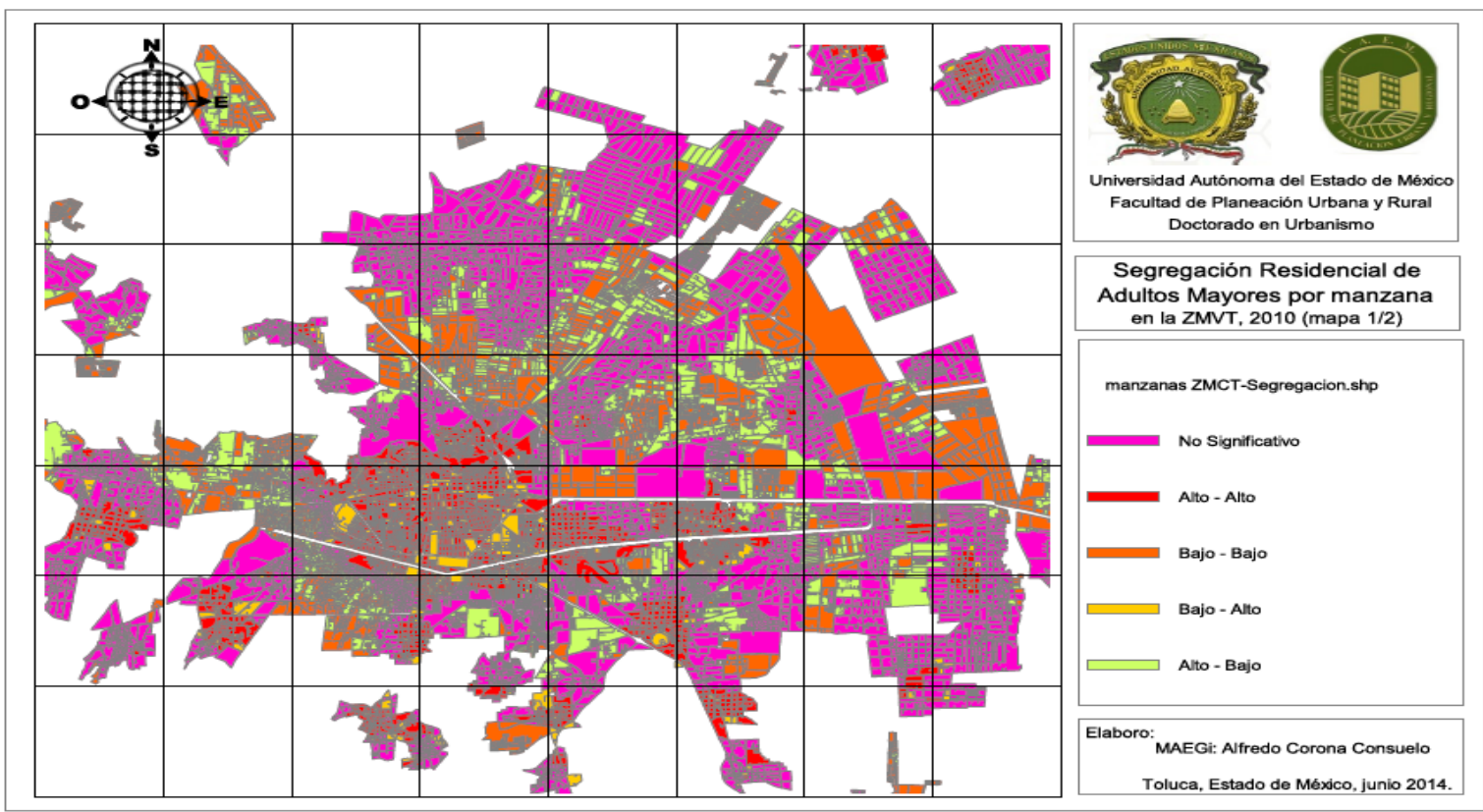

Figura 7. Mapa de Índice de Moran para la SRAM en ZMVT, 2010. Fuente: Elaboración propia sobre la base de datos de SCINCE 2010 (INEGI) y con ayuda de software GEODA y gv SIG.

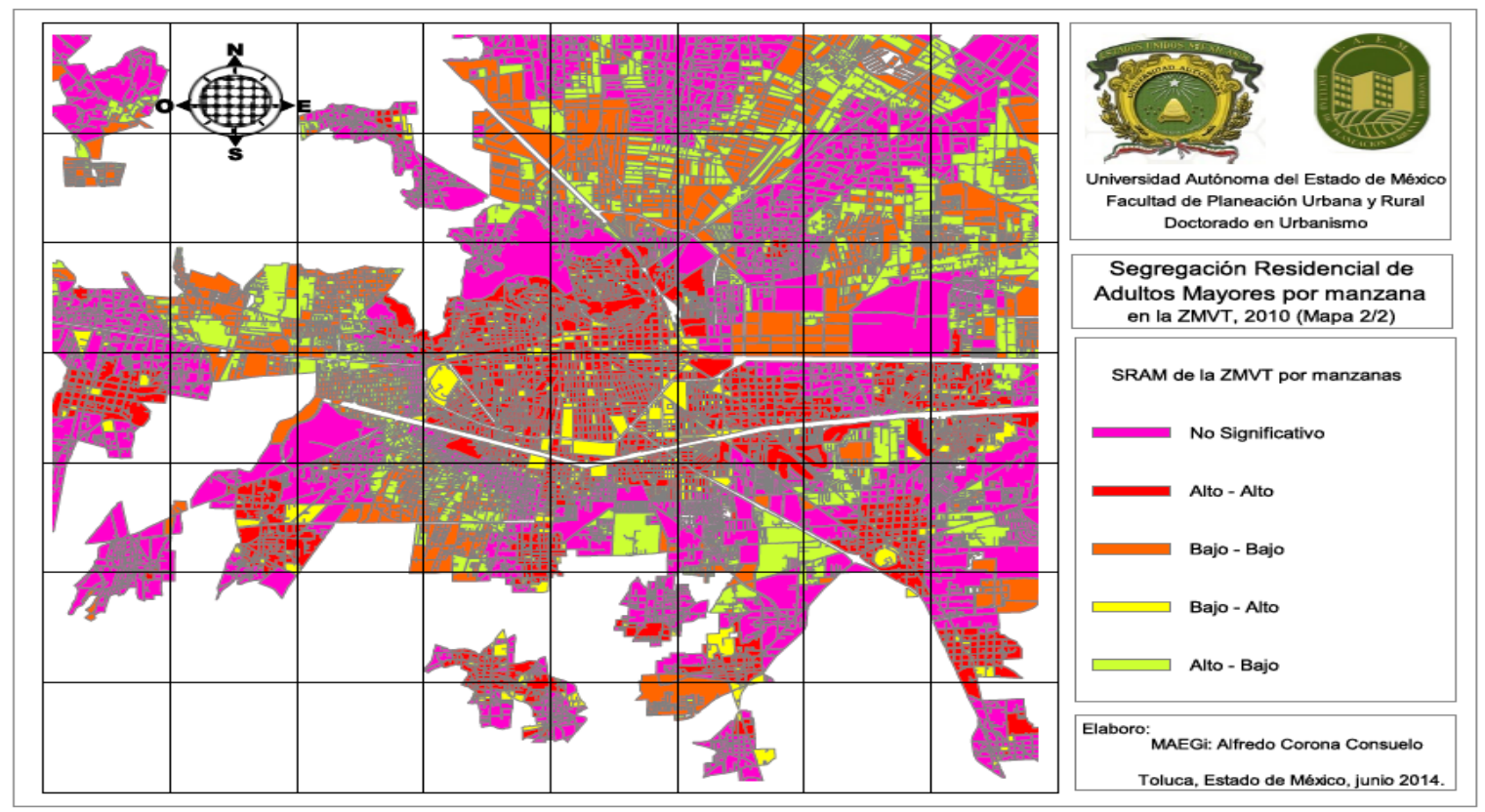

Figura 8. Mapa de Índice de Moran para la SRAM en ZMVT, 2010.

Fuente: Elaboración propia sobre la base de datos de SCINCE 2010 (INEGI) y con ayuda de software GEODA y gv SIG. 


\section{Conclusiones}

Respecto de este tema podemos observar que existe una Zona Metropolitana extensa que abarca 21 municipios, de los cuales dos son los que jerárquicamente adquieren mayor importancia (Toluca y Metepec), además de que conforman el centro urbano de esta Zona Metropolitana y tienen diferentes funciones.

Algunas de las conclusiones que pueden extraerse a partir del análisis realizado se despliegan en los siguientes párrafos.

La ciudad está fragmentada en una multiestructura socioeconómico residencial. Existe una extensión de zona habitacional en lo que abarca esta área metropolitana dominada por conjuntos habitacionales, lo cual beneficia la expansión de la mancha urbana y la conformación de la ciudad (Almandoz, 2006; Inzulza-Contardo, 2012; Jansochka, 2002), pero que sin embargo fragmenta la ciudad y muestra una multiestructura social, un mosaico urbano, de notables diferencias económicas (Borsdorf, 2013).

Así mismo, la población adulta mayor reside en el centro de la ciudad a pesar del cambio de funcionalidad de esta. En efecto, aún con el cambio de usos dentro de la ciudad y la variedad de funciones que esta adquiere, la población adulta mayor parece aguantar y soportar estos cambios funcionales. En cierta medida se puede suponer que debe influir en ello la idiosincrasia de esta población, así como también la comodidad de que en estas áreas se encuentran todos los servicios que requieren, lo que les permite vivir más cómodamente.

Por su parte, se ha constatado que la econometría regional (Geoeconometría) es una herramienta muy útil en análisis geográficos (caso específico Geografía Gerontológica), ya que esta y sus instrumentos de medición adquieren un cierto rango de importancia cuando se trata de estudiar datos espaciales.

Por último, se puede afirmar que las ciudades mexicanas viven en una tendencia a envejecer en el mediano y/o corto plazo. Pese a que se han realizado ya varios estudios acerca del tema del envejecimiento en el país, las medidas en apoyo para este grupo de población son muy pocas: las ciudades son cada vez más transitadas, ruidosas, inseguras y no se hable de contaminadas, por lo que debieran analizarse e implementarse mayores medidas para beneficiar a este grupo y confortarles las vida, tomándolos en consideración al momento de administrar y gestionar el desarrollo de los centros urbanos. 


\section{Referencias bibliográficas}

Almandoz, A. (2006). Urban planning and historiography in Latin America. Progress in Planning, 65(2), 81-123. https://doi.org/10.1016/j.progress.2006.02.002

Anselin, L. (1995). Local indicators of spatial association- LISA. Geographical analysis, 27(2), 93-115. $\quad$ https://doi.org/10.1111/j.15384632.1995.tb00338.x

Anselin, L., Rey S., \& Li, W. (2014). Metadata and provenance for spatial analysis: The case of spatial weights. International Journal of Geographical Information Science, 28(11), 2261-2280. https://doi.org/10.1080/13658816.2014.917313

Borja, J. y Castells, M. (2000). Local y global. La gestión de las ciudades en la era de la información. México: Taurus.

Borsdorf, A. (2003). ¿Cómo modelar el desarrollo y la dinámica de la ciudad latinoamericana? Eure, 86, 37-49. https://doi.org/10.4067/s025071612003008600002

Checa-Olmos y Arjona Garrido, A. (2007). Factores explicativos de la segregación residencial de los inmigrantes en Almería. Revista Internacional de Sociología (RIS), 65(48), 173-200. https://doi.org/10.3989/ris.2007.i48.73

Consejo Nacional de Población, CONAPO. (2013). Proyecciones 2030, México D.F. Recuperado de http://www.conapo.gob.mx

Corona, C. A., Garrocho, R., y Campos A., J. (2014). Segregación residencial de adultos mayores en ciudades de México: un análisis de autocorrelación espacial. Geografía y Sistemas de Información Geográfica (GEOSIG-UNLU, Luján), 6(6), 100-119. Recuperado de http://www.gesig-proeg.com.ar/documentos/revista-geosig/2014/07CORONA_ETAL_1_2014.pdf

Garrocho, C. (2013). Dinámica de las ciudades de México en el siglo XXI. Estado de México: ONU, Fondo de Población-Conapo-El Colegio Mexiquense.

Garrocho, C. y Campos, J. (2005). La población adulta mayor en el área metropolitana de Toluca, 1990-2000. Papeles de Población, 11(45), 71106. Recuperado de http://www.redalyc.org/pdf/112/11204505.pdf 
Instituto Nacional de Geografía, INEGI. (2010). Censo de Población y Vivienda 2010. Consulta interactiva de datos. Recuperado de http://www.inegi.org.mx/est/contenidos/proyectos/ccpv/cpv2010/

Inzulza-Contardo, J. (2012). 'Latino Gentrification'?: Focusing on physical and socioeconomic patterns of change in Latin American inner cities. Urban Studies, 49(10), https://doi.org/10.1177/0042098011423425

2085-2107.

Janoschka, M. (2002). El nuevo modelo de la ciudad latinoamericana: fragmentación y privatización. Eure, 85, 11-20. https://doi.org/10.4067/s0250-71612002008500002

Moreno, R. y Vayá, E. (2000). Técnicas econométricas para el tratamiento de datos espaciales: la econometría espacial. Barcelona: UB 44 Manuals, Edicions Universitat de Barcelona.

Ordorica, M. (2012). ¿Como aprovechar el éxito de la política de población del último cuarto de siglo XXI para enfrentar los nuevos retos demográficos del XXI? Papeles de Población, 18(74), 9-15. Recuperado de http://www.redalyc.org/pdf/112/11225471002.pdf

Ortiz, J. y Morales, S. (2002). Impacto socioespacial de las migraciones intraurbanas en entidades de centro y de nuevas periferias del Gran Santiago. Eure, 28(85), 171-185. https://doi.org/10.4067/s025071612002008500009

Prieto, M. B. (2010). La segregación socio-residencial en ciudades intermedias el caso de Bahía Blanca-Argentina. Secretaría de Ciencia y Tecnología de la Universidad Nacional del Sur, Argentina.

Rodríguez, J. (2008a). Movilidad cotidiana, desigualdad social y segregación residencial en cuatro metrópolis de América Latina, Eure, 34(103), 4971. https://doi.org/10.4067/s0250-71612008000300003

Rodríguez, J. (2008b). Dinámica sociodemográfica metropolitana y segregación residencial: ¿qué aporta la CASEN 2006? Revista de Geografía Norte Grande, $\quad 41$, 81-102. 34022008000300005 